\title{
MECANISMOS DE TRANSFERÊNCIA DE MASSA NA DESIDRATAÇÃO OSMÓTICA DE GOIABA EM SOLUÇÕES DE SACAROSE, SUCRALOSE E AÇÚÇAR INVERTIDO
}

\author{
VALÉRIA A. V. QUEIROZ ${ }^{1}$, PEDRO A. BERBERT ${ }^{2}$, MARÍLIA A. B. MOLINA ${ }^{3}$, \\ GERALDO A. GRAVINA ${ }^{4}$, LUCIANO R. QUEIROZ ${ }^{5}$
}

RESUMO: O objetivo deste trabalho foi avaliar o efeito da concentração de soluções de sacarose, sucralose e açúcar invertido sobre a cinética da desidratação osmótica de pedaços de goiaba. Frações de $1 / 12$ do fruto foram imersas em soluções de sacarose a $0,5 \mathrm{e} 0,4 \mathrm{~g} \mathrm{~mL}^{-1}$, de sacarose a $0,3 \mathrm{~g} \mathrm{~mL}^{-1}+$ sucralose a $0,2 \mathrm{~g} \mathrm{~L}^{-1}$ e em xarope de açúcar invertido, a $50{ }^{\circ} \mathrm{C}$, por $2 \mathrm{~h}$, sob agitação de 60 min. A solução de açúcar invertido promoveu maior perda de água e redução de massa nas amostras de goiaba submetidas à desidratação osmótica. $\mathrm{O}$ melhor desempenho foi obtido para o tratamento em solução de sacarose a $0,4 \mathrm{~g} \mathrm{~mL}^{-1}$, com perda de água e redução de massa semelhantes aos valores obtidos na imersão em solução de sacarose a $0,5 \mathrm{~g} \mathrm{~mL}^{-1}$ e ganho de sólidos similar ao observado em solução de sacarose a $0,3 \mathrm{~g} \mathrm{~mL}^{-1}$.

PALAVRAS-CHAVE: Psidium guajava, desidratação por imersão-impregnação.

\section{MASS TRANSFER MECHANISMS DURING THE OSMOTIC DEHYDRATION OF GUAVA IN SUCROSE, SUCRALOSE AND INVERTED SUGAR SOLUTIONS}

\begin{abstract}
The present work aimed at investigating the effect of sucrose, sucralose and inverted sugar solutions on the kinetics of osmotic dehydration of guava pieces. The fruits were cut in twelfths and immersed in sucrose solutions at 0.5 and $0.4 \mathrm{~g} \mathrm{~mL}^{-1}$, of sucrose at $0.3 \mathrm{~g} \mathrm{~mL}^{-1}+$ sucralose at $0.2 \mathrm{~g} \mathrm{~L}^{-1}$ and in inverted sugar syrup for $2 \mathrm{~h}$ at $50{ }^{\circ} \mathrm{C}$, under agitation of $60 \mathrm{~min}$. The undiluted inverted sugar solution promoted the highest levels of water loss and weight reduction in osmo-dehydrated guava pieces. The best overall performance was achieved by immersing guava pieces in sucrose solutions at $0.4 \mathrm{~g} \mathrm{~mL}^{-1}$ which led to water loss and mass reduction of similar values attained with sucrose solutions at $0.5 \mathrm{~g} \mathrm{~mL}^{-1}$, whereas maintaining the same level of solids gain achieved with sucrose solutions at $0.3 \mathrm{~g} \mathrm{~mL}^{-1}$.
\end{abstract}

KEYWORDS: Psidium guajava, dewatering and impregnation soaking.

\section{INTRODUÇÃO}

A desidratação osmótica (DO), também denominada desidratação por imersão-impregnação, tem sido utilizada como pré-tratamento no processamento de frutas por reduzir sua atividade de água, aumentar a estabilidade do produto e melhorar suas propriedades sensoriais, funcionais e nutricionais (TORREGGIANI \& BERTOLO, 2001). Pedaços de frutas são imersos em soluções hipertônicas, usando principalmente a sacarose como agente osmótico, resultando em fluxos de água e de substâncias solúveis da fruta para o meio e, em sentido contrário, de soluto para o produto. A cinética do processo é geralmente avaliada em termos de perda de água $(\omega)$, ganho de sólidos $(\delta)$ e perda de massa $(\mu)$ (SILVEIRA et al., 1996).

O fenômeno de transferência de massa que acontece entre o produto e o meio osmótico é amplamente afetado pelas variáveis do processo, as quais estão associadas tanto à natureza da matéria-prima quanto às condições operacionais empregadas. Interferem no desempenho da desidratação a espécie utilizada, a variedade, o grau de maturação, a forma e o tamanho do alimento

\footnotetext{
${ }^{1}$ Pesquisador A, Embrapa Milho e Sorgo, Sete Lagoas - MG, valeria@ cnpms.embrapa.br.

${ }^{2}$ Eng ${ }^{\mathrm{O}}$ Agrícola, Prof. Adjunto, UENF, Campos dos Goytacazes - RJ, pberbert@uenf.br.

${ }^{3}$ Eng $^{\mathrm{a}}$ de Alimentos, Prof. Adjunto, UENF, Campos dos Goytacazes - RJ, mberbert@uenf.br.

${ }^{4}$ Eng ${ }^{\mathrm{o}}$ Agrônomo, Prof. Adjunto, UENF, Campos dos Goytacazes - RJ, gravina@uenf.br.

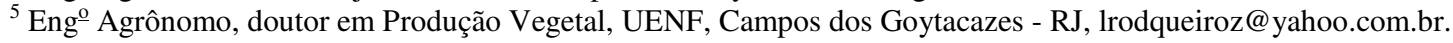

Recebido pelo Conselho Editorial em: 29-8-2008
}

Aprovado pelo Conselho Editorial em: 12-12-2009 
a ser desidratado, assim como a composição, a concentração e a temperatura da solução osmótica, o tempo de contato da fração sólida com o xarope, a razão entre a massa do produto (fruta) e a massa do meio (xarope) e a utilização ou não de pré-tratamentos. O resultado é a redução do teor de água com concomitante aumento da massa seca e mudança na composição química (DHINGRA et al., 2008).

A goiaba é considerada fruto climatérico, exibindo intenso metabolismo, com aumento típico da respiração e da produção de etileno durante o amadurecimento, sendo essa característica responsável pela sua alta perecibilidade (ALI \& LAZAN, 2001). A safra de goiabas concentra-se no período entre janeiro e fevereiro, havendo excedente não comercializado para consumo in natura, com consequente queda dos preços no varejo. Dessa forma, o processamento dos frutos excedentes da produção por meio da desidratação osmótica pode tornar-se importante alternativa para $o$ produtor que se dedica a essa cultura (BRUNINI et al., 2003).

São relativamente poucos os trabalhos a respeito da desidratação osmótica de goiaba, a maioria havendo sido realizado empregando-se apenas sacarose como agente osmótico (PEREIRA et al., 2004; PANADÉS et al., 2006; PANADÉS et al., 2008; PINO et al., 2008). QUEIROZ et al. (2007) avaliaram as características físico-químicas e sensoriais de goiabas submetidas à desidratação osmótica em soluções de sacarose e de açúcar invertido e posteriormente secadas por convecção, bem como avaliaram a estabilidade da cor dos frutos depois de 30; 60 e 90 dias de armazenamento sob refrigeração. QUEIROZ et al. (2008) avaliaram a qualidade nutricional de goiabas submetidas a esses mesmos processos e observaram reduções de 20 a $64 \%$ no teor de minerais ao final da desidratação osmótica e perdas não significativas de ácido ascórbico, exceto no tratamento com açúcar invertido sem diluição.

De acordo com DHINGRA et al. (2008), a composição e a concentração da solução osmótica são as principais variáveis que afetam o processo de transferência de massa na desidratação osmótica de frutas. Devido à complexidade da microestrutura dos tecidos das plantas, há necessidade de se estudarem as diferentes variáveis envolvidas no processo em cada espécie vegetal. Assim, apesar de já haver alguns trabalhos a respeito da DO de goiaba, informações sobre a influência do tipo e da concentração de diferentes soluções osmóticas sobre a perda de água, o ganho de sólidos e a redução de massa na DO dessa fruta são ainda inexistentes. Sendo assim, o objetivo deste trabalho foi avaliar o efeito da desidratação osmótica em soluções de sacarose, sacarose + sucralose e açúcar invertido sobre a perda de água, o ganho de sólidos e a redução de massa em pedaços de goiaba.

\section{MATERIAL E MÉTODOS}

Foram utilizadas 12 goiabas (Psidium guajava L.) da cultivar Pedro Sato, provenientes de pomar comercial localizado no município de Cachoeiras de Macacu - RJ, colhidas no estádio de maturação 2, com ângulo de cor $\left(\mathrm{h}^{\circ}\right)$ entre 113 e 116, o que caracteriza a casca como verde-clara. Foram escolhidos frutos que apresentavam uniformidade de tamanho e forma, além da ausência de injúrias. Os frutos selecionados foram acondicionados em bandejas plásticas e armazenados sob refrigeração, em câmaras do tipo B.O.D. a $7{ }^{\circ} \mathrm{C}$, pelo tempo necessário para atingir o estádio de maturação 3, caracterizado pela cor verde-amarelada da casca, com ângulo de cor entre 109 e 112.

Os frutos, depois de retirados do ambiente de armazenamento, foram lavados em água corrente e imersos em solução de detergente neutro a $1 \%$ por $15 \mathrm{~min}$, sendo posteriormente enxaguados em água corrente, sanitizados em solução de hipoclorito de sódio a 100 ppm por 15 min e secos com papel-toalha. Em seguida, foram descascados e cortados transversalmente, retirando-se as sementes e dividindo-se cada metade em outras seis partes, de modo a obterem-se 12 frações por fruto, num total de 144 frações.

A caracterização físico-química das goiabas in natura quanto aos parâmetros teor de água, massa, diâmetro longitudinal (DL), diâmetro equatorial (DE), relação DL/DE, espessura da polpa 
sólidos solúveis totais ( ${ }^{\circ}$ Brix), acidez total titulável, cor da casca e cor da polpa, foi feita de acordo com metodologia descrita em QUEIROZ et al. (2008).

Para evitar o escurecimento enzimático, as amostras foram sequencialmente branqueadas em vapor d'água por $1 \mathrm{~min}$, resfriadas em banho de gelo por $1 \mathrm{~min}$ (dentro de saco plástico) e imersas em solução de ácido ascórbico (1\%) por 5 min. Depois dessas etapas, foram retiradas duas frações de 1/12 de cada um dos 12 frutos. Uma das frações foi utilizada para a determinação dos teores de água e de sólidos solúveis do fruto in natura, e a outra fração foi utilizada com o mesmo propósito, porém para caracterizar a condição inicial (depois da imersão em ácido ascórbico) de cada fruto que viria a ser posteriormente utilizado na desidratação.

As 120 frações restantes (dez por fruto) foram submetidas à desidratação osmótica em soluções de sacarose a $0,5 \mathrm{~g} \mathrm{~mL}^{-1}$ (tratamento T1), sacarose a $0,4 \mathrm{~g} \mathrm{~mL}^{-1}$ (tratamento T2), sacarose a $0,3 \mathrm{~g} \mathrm{~mL}^{-1}+$ sucralose $0,2 \mathrm{~g} \mathrm{~mL}^{-1}$ (tratamento $\mathrm{T} 3$ ) e xarope de açúcar invertido sem diluição (tratamento T4). Foram utilizados sacarose em duas concentrações e açúcar invertido em uma concentração para avaliar o efeito da concentração e do tipo de soluto osmótico sobre o processo de transferência de massa em goiaba. Além de sacarose, utilizou-se a sucralose com o objetivo de obter um produto light (> 25\% de redução de calorias) com o mesmo nível de doçura do Tratamento T2 (sacarose $0,3 \mathrm{~g} \mathrm{~mL}^{-1}$ ), porém com $30 \%$ menos caloria, resultando no Tratamento T3. Para isso, no cálculo da massa do adoçante sucralose, foi considerada que a relação poder edulcorante de $1.000 \mathrm{~g}$ de sacarose corresponde àquele de 1,67 $\mathrm{g}$ de sucralose.

O teor de sólidos solúveis totais (SST, ${ }^{\circ} \mathrm{Brix}$ ) nas soluções foi determinado com refratômetro portátil Atago, modelo HSR 500, de acordo com metodologia proposta pela AOAC (1990a). O processo foi realizado em incubadoras-agitadoras de bancada (B. Braun Biotech, modelo Certomat U/HK) de movimento orbital, com frequência de agitação de $60 \mathrm{~min}$, a $50{ }^{\circ} \mathrm{C}$ por $2 \mathrm{~h}$, empregando-se relação fruta: xarope de 1: 10 (em massa). Para cada tipo de solução osmótica, foram utilizados seis béqueres de $1.000 \mathrm{~mL}$. Em três desses béqueres, foram colocadas sete frações de três goiabas (21 frações por tratamento), ou seja, para cada solução osmótica, foram utilizados três frutos, caracterizando três repetições por tratamento. Essas sete frações foram utilizadas para a determinação da cinética do processo (perda de água e ganho de sólidos) aos 5; 15; 30; 45; 60; 90 e 120 min. de imersão.

Depois de retiradas da solução osmótica, as amostras foram enxaguadas com água destilada para a remoção do filme de açúcar aderido à superfície e envolvidas suavemente em papel absorvente para a retirada do excesso de água. A preparação das amostras para a determinação dos teores de água e de sólidos solúveis, em cada intervalo de tempo e para cada tratamento, foi feita utilizando-se de triturador Tecnal, modelo Turratec TE-102. Depois de pesadas em balança analítica Sartorius, modelo BL 210S, as amostras foram secadas em estufa a vácuo Nova Ética, modelo $440 \mathrm{D}$ a $70^{\circ} \mathrm{C}$, sob pressão de $13,3 \mathrm{kPa}$ (AOAC, 1990b). As três frações restantes por fruto (nove frações por tratamento) foram colocadas em outro béquer de $1.000 \mathrm{~mL}$, contendo as respectivas soluções osmóticas, sendo identificadas com pinos coloridos e utilizadas para a determinação da redução de massa. Essas amostras, depois de retiradas da solução, foram submetidas ao mesmo procedimento descrito anteriormente, ou seja, enxágue e remoção da água superficial, antes do procedimento de pesagem; no entanto, depois de pesadas, ao final dos intervalos de tempo preestabelecidos, cada fração era recolocada na solução osmótica.

A sacarose foi adquirida no mercado local, e o xarope de açúcar invertido, com denominação comercial de Gludex 216 e com taxa de inversão $\geq 90 \%$, foi fornecido pela Dulcini S.A. - SP. O edulcorante em pó sucralose (1,6-dicloro-1,6-dideoxi- $\beta$-D-frutofuranosil-4-cloro-4-deoxi- $\alpha$-Dgalacto-piranosídeo) foi fornecido pela empresa Attivos Magisttrais. As soluções osmóticas foram preparadas com água destilada e estocadas em B.O.D. a $7^{\circ} \mathrm{C}$ até o momento de sua utilização.

Para a obtenção dos valores da perda de água $(\omega)$, do ganho de sólidos $(\delta)$ e da redução de massa $(\mu)$ ao longo da desidratação osmótica, determinaram-se experimentalmente os teores de água e de sólidos totais das amostras em cada um dos intervalos de tempo, empregando-se o método 
gravimétrico. Os cálculos de $\mu, \omega$ e $\delta$ foram feitos de acordo com as eqs.(1), (2) e (3), em que $\mathrm{m}_{\mathrm{i}}$ massa inicial da amostra de goiaba, $\mathrm{g} ; \mathrm{m}_{\mathrm{t}}$ - massa da amostra no tempo $\mathrm{t}, \mathrm{g} ; \mathrm{X}_{\mathrm{ai}}$ - fração de água na amostra no tempo inicial, adimensional; $X_{\text {at }}$ - fração de água na amostra no tempo $t$, adimensional; $\mathrm{X}_{\mathrm{si}}$ - fração de sólidos totais da amostra no tempo inicial, adimensional, e $\mathrm{X}_{\mathrm{st}}$ - fração de sólidos totais da amostra no tempo $t$, adimensional (SILVEIRA et al., 1996).

$$
\begin{aligned}
& \mu=\left(\frac{\mathrm{m}_{\mathrm{i}}-\mathrm{m}_{\mathrm{t}}}{\mathrm{m}_{\mathrm{i}}}\right) 100 \\
& \omega=\left(\frac{\mathrm{m}_{\mathrm{i}} \mathrm{X}_{\mathrm{ai}}-\mathrm{m}_{\mathrm{t}} \mathrm{X}_{\mathrm{at}}}{\mathrm{m}_{\mathrm{i}}}\right) 100 \\
& \delta=\left(\frac{\mathrm{m}_{\mathrm{i}} \mathrm{X}_{\mathrm{st}}-\mathrm{m}_{\mathrm{t}} \mathrm{X}_{\mathrm{si}}}{\mathrm{m}_{\mathrm{i}}}\right) 100
\end{aligned}
$$

O experimento foi planejado de acordo com o delineamento em blocos casualizados, com arranjo fatorial (4 soluções de desidratação x 7 tempos de imersão), com três repetições (três frutos). Os valores obtidos foram avaliados por análise de variância, depois da verificação da normalidade dos dados e da homogeneidade das variâncias dos tratamentos pelos testes de Lilliefors e Bartlett, respectivamente, a 5\% de probabilidade. Para as características cuja interação entre solução de desidratação e tempo de imersão foi significativa pelo teste " $F$ " da ANOVA, promoveram-se os desdobramentos. As médias das variáveis em cada solução osmótica ao longo do tempo de imersão foram avaliadas por análise de regressão. $\mathrm{O}$ melhor modelo de regressão foi escolhido pelo coeficiente de determinação $\left(\mathrm{r}^{2}\right)$ e pela significância dos coeficientes da regressão $\left(\hat{\beta}_{1}\right)$, que foram testados pelo teste $t$ de Student. Para a comparação das médias entre as soluções dentro de cada tempo, foi utilizado o teste de Tukey, a 5\% de probabilidade. As análises estatísticas foram realizadas empregando-se o aplicativo computacional SAEG (Sistema para Análises Estatísticas e Genéticas), versão 9.0.

\section{RESULTADOS E DISCUSSÃO}

\section{Caracterização físico-química do fruto in natura e das soluções osmóticas}

A caracterização físico-química das goiabas in natura (em base úmida) no estádio três de maturação encontra-se na Tabela 1. As características dos frutos estão de acordo com os valores observados por AZZOLINI et al. (2004) e aqueles apresentados na Tabela Brasileira de Composição de Alimentos (TACO, 2006). Foram obtidos os seguintes valores médios para os teores de SST das soluções ao longo da desidratação: sacarose $0,3 \mathrm{~g} \mathrm{~mL}^{-1}+$ sucralose $0,2 \mathrm{~g} \mathrm{~L}^{-1}$ (26 ${ }^{\circ}$ Brix); sacarose $0,4 \mathrm{~g} \mathrm{~mL}^{-1}$ (32 ${ }^{\circ}$ Brix); sacarose $0,5 \mathrm{~g} \mathrm{~mL}^{-1}$ (40 ${ }^{\circ}$ Brix $)$ e xarope de açúcar invertido (64 ${ }^{\circ}$ Brix).

TABELA 1. Características físico-químicas de goiabas $c v$ Pedro Sato no estádio de maturação três. Physical chemistry characterization of guava cv Pedro Sato in the the stage of ripening.

\begin{tabular}{lclc}
\hline \multicolumn{1}{c}{ Variável } & Valor Médio & \multicolumn{1}{c}{ Variável } & Valor Médio \\
\hline Teor de água (\% b.u.) & $86,7 \pm 1,1$ & SST $\left({ }^{\circ}\right.$ Brix $)$ & $8,6 \pm 1,3$ \\
Massa (g) & $185,5 \pm 37,3$ & ATT* $(\%$ ácido ascórbico) & $0,46 \pm 0,05$ \\
Diâmetro longitudinal - DL (cm) & $7,9 \pm 0,8$ & SST/ATT & 18,7 \\
Diâmetro equatorial - DE (cm) & $6,8 \pm 1,0$ & pH & $4,03 \pm 0,23$ \\
DL/DE & 1,2 & Cor da casca - parâmetro ${ }^{\circ}$ & $106,50 \pm 3,14$ \\
Espessura da polpa $(\mathrm{cm})$ & $0,83 \pm 0,17$ & Cor da polpa - parâmetro C** & $33,56 \pm 3,60$ \\
\hline
\end{tabular}

*ATT - acidez total titulável; ** C - cromaticidade. 


\section{Análise global da cinética de desidratação}

Os valores experimentais e estimados da perda de água $(\omega)$, do ganho de sólidos $(\delta)$ e da redução de massa $(\mu)$, dos pedaços de goiaba, em função do tempo de imersão e da desidratação, para os quatro tipos de solução osmótica empregados, são mostrados nas Figuras 1; 2 e 3, respectivamente. As equações de regressão e seus respectivos coeficientes de determinação para essas três variáveis que caracterizam a cinética da desidratação osmótica encontram-se na Tabela 2.

As mudanças observadas nos valores de declividade das curvas apresentadas nas Figuras 1;2 e 3, permitiram distinguir duas fases distintas para o comportamento dos parâmetros $\omega, \delta$ e $\mu$ em função do tempo de desidratação. Na primeira fase, a perda de água, o ganho de sólidos e a redução de massa ocorreram de forma intensa nos 30 minutos iniciais do processo. Na fase seguinte, os fluxos de massa passaram a decrescer gradualmente, tendendo ao equilíbrio a partir de $1 \mathrm{~h}$ e 30 , com exceção do tratamento com açúcar invertido, que continuou apresentando perda de água, porém em taxas menos acentuadas, até o final do processo. Esses resultados estão em conformidade com os relatados por PANADÉS et al. (2006) e KHOYI \& HESARI (2007), na desidratação de goiaba e damasco, respectivamente. DIONELLO et al. (2007), de forma semelhante, observaram que, na desidratação osmótica de abacaxi, os parâmetros que caracterizam a transferência de massa deixaram de apresentar diferenças significativas depois de $45 \mathrm{~min}$ de imersão. Entretanto, ANDRADE et al. (2007) mostraram que os fluxos de massa entre pedaços de jenipapo e soluções de sacarose a 30; 50 e 70\% começaram a reduzir consideravelmente depois de $2 \mathrm{~h}$ de desidratação osmótica, atingindo o equilíbrio apenas às 12; 24 e $32 \mathrm{~h}$ de imersão, respectivamente. Os autores argumentaram que esse tempo mais longo deveu-se, provavelmente, ao tipo de tecido dessa fruta, mais permeável ao soluto osmótico.

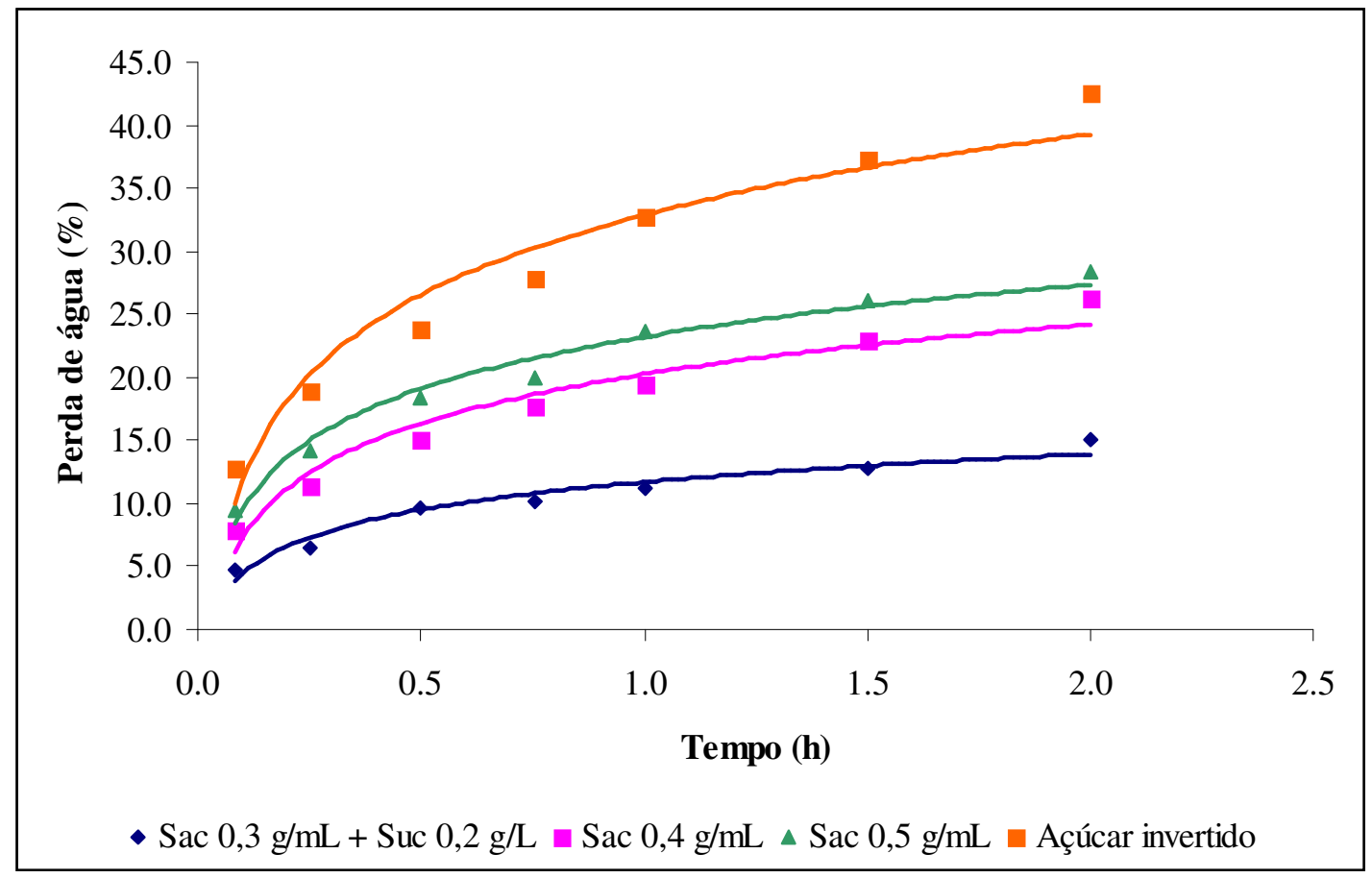

FIGURA 1. Perda de água $(\omega)$ dos pedaços de goiaba em função do tempo de desidratação osmótica, em soluções de sacarose a 0,5 e $0,4 \mathrm{~g} \mathrm{~mL}^{-1}$, de sacarose a $0,3 \mathrm{~g} \mathrm{~mL}^{-1}+$ sucralose $0,2 \mathrm{~g} \mathrm{~L}^{-1}$ e xarope de açúcar invertido sem diluição, a $50{ }^{\circ} \mathrm{C}$ por $2 \mathrm{~h}$. Water loss $(\omega)$ in guava pieces as a function of osmo-dehydration time in sucrose solutions at 0.5 and $0.4 \mathrm{~g} \mathrm{~mL}^{-1}$, in sucrose at $0.3 \mathrm{~g} \mathrm{~mL}^{-1}+$ sucralose at $0.2 \mathrm{~g} \mathrm{~mL}^{-1}$, in undiluted inverted sugar syrup, at $50^{\circ} \mathrm{C}$ for $2 \mathrm{~h}$. 


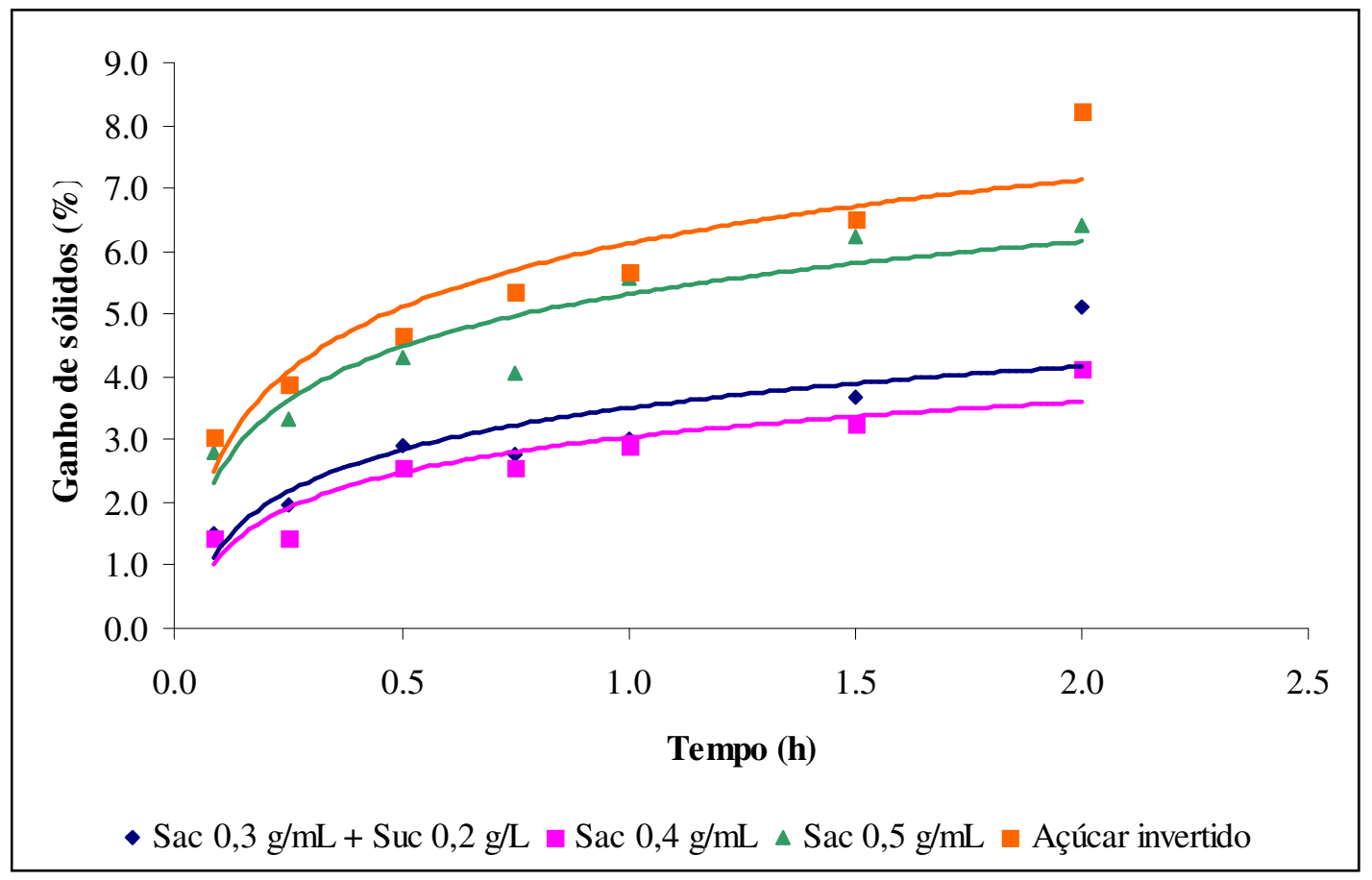

FIGURA 2. Ganho de sólidos $(\delta)$ dos pedaços de goiaba em função do tempo de desidratação osmótica, em soluções de sacarose a 0,5 e $0,4 \mathrm{~g} \mathrm{~mL}^{-1}$, de sacarose a $0,3 \mathrm{~g} \mathrm{~mL}^{-1}+$ sucralose $0,2 \mathrm{~g} \mathrm{~L}^{-1}$ e xarope de açúcar invertido sem diluição, a $50{ }^{\circ} \mathrm{C}$ por $2 \mathrm{~h}$. Solids gain $(\delta)$ in guava pieces as a function of osmo-dehydration time in sucrose solutions at 0.5 and $0.4 \mathrm{~g} \mathrm{~mL}^{-1}$, in sucrose at $0.3 \mathrm{~g} \mathrm{~mL}^{-1}+$ sucralose at $0.2 \mathrm{~g} \mathrm{~mL}^{-1}$, in undiluted inverted sugar syrup, at $50^{\circ} \mathrm{C}$ for $2 \mathrm{~h}$.

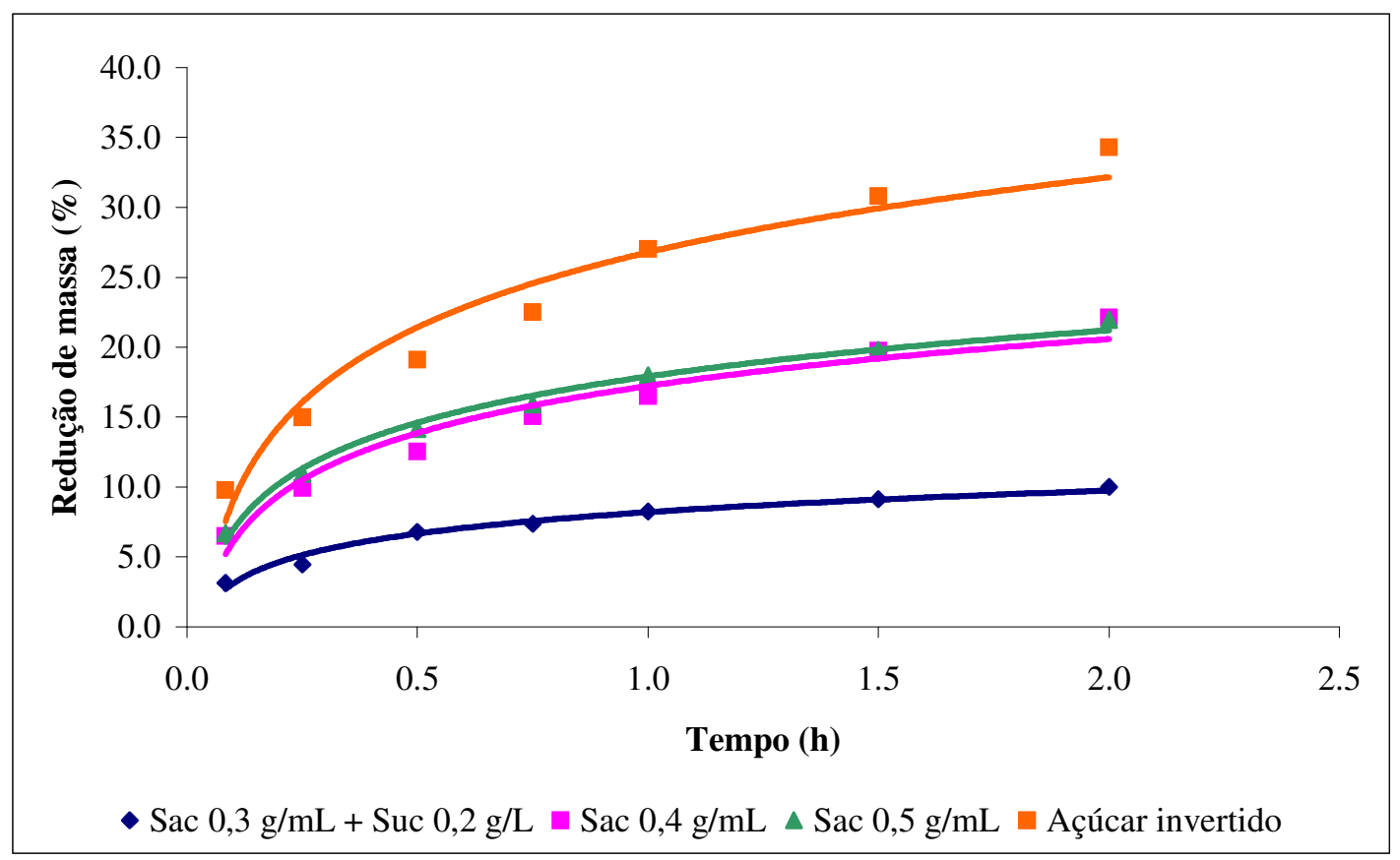

FIGURA 3. Redução de massa $(\mu)$ dos pedaços de goiaba em função do tempo de desidratação osmótica, em soluções de sacarose a 0,5 e $0,4 \mathrm{~g} \mathrm{~mL}^{-1}$, de sacarose a $0,3 \mathrm{~g} \mathrm{~mL}^{-1}+$ sucralose $0,2 \mathrm{~g} \mathrm{~L}^{-1}$ e xarope de açúcar invertido sem diluição, a $50^{\circ} \mathrm{C}$ por $2 \mathrm{~h}$. Mass reduction $(\mu)$ in guava pieces as a function of osmo-dehydration time in sucrose solutions at 0.5 and $0.4 \mathrm{~g} \mathrm{~mL}^{-1}$, in sucrose at $0.3 \mathrm{~g} \mathrm{~mL}^{-1}+$ sucralose at $0.2 \mathrm{~g} \mathrm{~mL}^{-1}$, in undiluted inverted sugar syrup, at $50^{\circ} \mathrm{C}$ for $2 \mathrm{~h}$. 
TABELA 2. Equações de regressão e coeficientes de determinação $\left(\mathrm{r}^{2}\right)$ para perda de água $(\omega, \%)$, ganho de sólidos $(\delta, \%)$ e redução de massa $(\mu, \%)$ em função do tempo (h) de desidratação osmótica de pedaços de goiaba em soluções de sacarose a 0,5 e $0,4 \mathrm{~g} \mathrm{~mL}^{-1}$, de sacarose a $0,3 \mathrm{~g} \mathrm{~mL}^{-1}+$ sucralose $0,2 \mathrm{~g} \mathrm{~L}^{-1}$ e xarope de açúcar invertido sem diluição, a $50{ }^{\circ} \mathrm{C}$. Estimated regression equations and their coefficients of determination for water loss $(\omega, \%)$, solids gain $(\delta, \%)$, and weight reduction $(\mu$, $\%$ ) as a function of osmo-dehydration time of guava pieces in sucrose solutions at 0.5 and $0.4 \mathrm{~g} \mathrm{~mL}^{-1}$, of sucrose at $0.3 \mathrm{~g} \mathrm{~mL}^{-1}+$ sucralose at $0.2 \mathrm{~g} \mathrm{~mL}^{-1}$, of undiluted inverted sugar syrup, at $50^{\circ} \mathrm{C}$.

\begin{tabular}{|c|c|c|c|}
\hline $\begin{array}{l}\text { Parâmetro da Cinética da } \\
\text { Desidratação Osmótica }\end{array}$ & Tratamento (solução osmótica) & Equação de Regressão & $r^{2}$ \\
\hline \multirow{4}{*}{ Perda de água $(\omega)$} & Sac $0,5 \mathrm{~g} \mathrm{~mL}^{-1}$ & $\hat{\omega}=5,978 \ln (\mathrm{t})^{* *}+23,224$ & 0,9797 \\
\hline & Sac $0,4 \mathrm{~g} \mathrm{~mL}^{-1}$ & $\hat{\omega}=5,661 \ln (\mathrm{t})^{* *}+20,266$ & 0,9513 \\
\hline & Sac $0,3 \mathrm{~g} \mathrm{~mL}^{-1}+$ Sucralose $0,2 \mathrm{~g} \mathrm{~L}^{-1}$ & $\hat{\omega}=3,172 \ln (\mathrm{t})^{* *}+11,708$ & 0,9523 \\
\hline & Açúcar invertido & $\hat{\omega}=9,201 \ln (\mathrm{t})^{* *}+32,907$ & 0,9481 \\
\hline \multirow{4}{*}{ Ganho de sólidos $(\delta)$} & Sac $0,5 \mathrm{~g} \mathrm{~mL}^{-1}$ & $\hat{\delta}=1,218 \ln (\mathrm{t})^{* *}+5,321$ & 0,8918 \\
\hline & $\mathrm{Sac} 0,4 \mathrm{~g} \mathrm{~mL}^{-1}$ & $\hat{\delta}=0,812 \ln (\mathrm{t})^{* *}+3,042$ & 0,8550 \\
\hline & Sac $0,3 \mathrm{~g} \mathrm{~mL}^{-1}+$ Sucralose $0,2 \mathrm{~g} \mathrm{~L}^{-1}$ & $\hat{\delta}=0,956 \ln (\mathrm{t})^{* *}+3,507$ & 0,8046 \\
\hline & Açúcar invertido & $\hat{\delta}=1,463 \ln (\mathrm{t})^{* *}+6,123$ & 0,8814 \\
\hline \multirow{4}{*}{ Redução de massa $(\mu)$} & Sac $0,5 \mathrm{~g} \mathrm{~mL}^{-1}$ & $\hat{\mu}=4,760 \ln (\mathrm{t})^{* *}+17,903$ & 0,9901 \\
\hline & Sac $0,4 \mathrm{~g} \mathrm{~mL}^{-1}$ & $\hat{\mu}=4,849 \ln (\mathrm{t})^{* *}+17,224$ & 0,9574 \\
\hline & Sac $0,3 \mathrm{~g} \mathrm{~mL}^{-1}+$ Sucralose $0,2 \mathrm{~g} \mathrm{~L}^{-1}$ & $\hat{\mu}=2,217 \ln (\mathrm{t})^{* *}+8,200$ & 0,9788 \\
\hline & Açúcar invertido & $\hat{\mu}=7,738 \ln (\mathrm{t})^{* *}+26,784$ & 0,9537 \\
\hline
\end{tabular}

**significativo $1 \%$ de probabilidade, pelo teste t.

\section{Perda de água}

As médias da percentagem de perda de água $(\omega)$ dos pedaços de goiaba entre as diferentes soluções osmóticas empregadas no processo, dentro de cada tempo, encontram-se na Tabela 3. Observa-se que, em todos os tempos amostrados, a perda de água foi significativamente menor nas amostras submetidas à solução de sacarose $0,3 \mathrm{~g} \mathrm{~mL}^{-1}+$ sucralose $0,2 \mathrm{~g} \mathrm{~L}^{-1}$ e maior (exceto no tempo de $0,1 \mathrm{~h}$ ) naquelas imersas em solução de açúcar invertido, possivelmente, devido ao efeito osmótico mais acentuado dessa última solução, o que resultou em maior fluxo de massa da fruta para o meio. $\mathrm{O}$ açúcar invertido contém os monossacarídeos glicose e frutose que exercem maior efeito osmótico que a sacarose devido a sua menor massa molecular (praticamente a metade), existindo, portanto, praticamente o dobro de moléculas de glicose que de sacarose na solução (KARATHANOS et al., 1995). Além disso, o açúcar invertido, por conter moléculas menores (glicose e frutose) comparado à sacarose, penetra mais nos tecidos da fruta e forma uma camada menos concentrada de açúcar na periferia das mesmas, com menor chance de cristalização, permitindo, assim, maior saída de água (RIVA et al., 2005).

Para soluções de sacarose $0,4 \mathrm{~g} \mathrm{~mL}^{-1}$ e $0,5 \mathrm{~g} \mathrm{~mL}^{-1}$, não houve diferença significativa para perda de água entre as amostras. Ao final de $2 \mathrm{~h}$ de imersão, verificou-se que a perda de água aumentou de 15,11 para $28,36 \%$, quando a concentração da solução aumentou de 0,3 para $0,5 \mathrm{~g} \mathrm{~mL}^{-1}$ de sacarose (a sucralose não possui efeito osmótico), ou seja, de 26 para $40^{\circ}$ Brix. FERRARI et al. (2005), ao estudarem a cinética da transferência de massa de melão desidratado osmoticamente, a 30 e a $40^{\circ} \mathrm{C}$, em soluções de sacarose a $40 ; 50$ e $60{ }^{\circ}$ Brix, verificaram aumento da perda de água à medida em que se aumentou a concentração das soluções. PARK et al. (2003) também relataram que a perda de água aumentou com o aumento da concentração do xarope na desidratação de cubos de pera em soluções de sacarose entre 40 e $70{ }^{\circ}$ Brix; o valor de $\omega$ para o tratamento com solução a $70{ }^{\circ}$ Brix foi de cerca de $30 \%$, similar, portanto, ao encontrado neste 
trabalho $(\omega=28 \%)$ para a solução de sacarose a $0,5 \mathrm{~g} \mathrm{~mL}^{-1}$ (40 ${ }^{\circ}$ Brix $)$ em $2 \mathrm{~h}$ de desidratação, mostrando, assim, maior eficiência na perda de água em goiaba do que em pera, para as geometrias e dimensões avaliadas nos respectivos trabalhos.

TABELA 3. Perda de água (\%) dos pedaços de goiaba em função do tempo de desidratação osmótica, em soluções de sacarose a $0,3 \mathrm{~g} \mathrm{~mL}^{-1}+$ sucralose $0,2 \mathrm{~g} \mathrm{~L}^{-1}$, sacarose a $0,5 \mathrm{e}$ $0,4 \mathrm{~g} \mathrm{~mL}^{-1}$ e xarope de açúcar invertido sem diluição. Water loss $(\boldsymbol{\omega})$ in guava pieces as a function of osmo-dehydration time in sucrose at $0.3 \mathrm{~g} \mathrm{~mL}^{-1}+$ sucralose at $0.2 \mathrm{~g} \mathrm{~mL}^{-1}$, in sucrose solutions at 0.5 and $0.4 \mathrm{~g} \mathrm{~mL}^{-1}$ and in undiluted inverted sugar syrup.

\begin{tabular}{ccccc}
\hline \multirow{2}{*}{ Tempo $(\mathrm{h})$} & \multicolumn{4}{c}{ Perda de água $(\%)$} \\
\cline { 2 - 5 } & Sac 0,3 $\mathrm{g} \mathrm{mL}^{-1}+$ Suc $0,2 \mathrm{~g} \mathrm{~L}^{-1}$ & ${\text { Sac } 0,4 \mathrm{~g} \mathrm{~mL}^{-1}}$ & Sac $0,5 \mathrm{~g} \mathrm{~mL}^{-1}$ & Açúcar invertido \\
\hline 0,1 & $4,65 \mathrm{C}$ & $7,91 \mathrm{~B}$ & $9,48 \mathrm{AB}$ & $12,82 \mathrm{AB}$ \\
0,3 & $6,42 \mathrm{C}$ & $11,36 \mathrm{~B}$ & $14,25 \mathrm{~B}$ & $18,88 \mathrm{~A}$ \\
0,5 & $9,70 \mathrm{C}$ & $15,08 \mathrm{~B}$ & $18,47 \mathrm{~B}$ & $23,75 \mathrm{~A}$ \\
0,8 & $10,11 \mathrm{C}$ & $17,61 \mathrm{~B}$ & $19,97 \mathrm{~B}$ & $27,85 \mathrm{~A}$ \\
1,0 & $11,24 \mathrm{C}$ & $19,39 \mathrm{~B}$ & $23,56 \mathrm{~B}$ & $32,69 \mathrm{~A}$ \\
1,5 & $12,80 \mathrm{C}$ & $23,00 \mathrm{~B}$ & $26,05 \mathrm{~B}$ & $37,29 \mathrm{~A}$ \\
2,0 & $15,11 \mathrm{C}$ & $26,26 \mathrm{~B}$ & $28,36 \mathrm{~B}$ & $42,52 \mathrm{~A}$ \\
\hline
\end{tabular}

Valores seguidos de mesma letra maiúscula na linha não diferem entre si, a 5\% de probabilidade, pelo teste de Tukey.

\section{Ganho de sólidos}

As médias da percentagem de ganho de sólidos $(\boldsymbol{\delta})$ dos pedaços de goiaba entre as diferentes soluções osmóticas empregadas no processo, dentro de cada tempo, encontram-se na Tabela 4. Para essa variável, não houve interação significativa entre o tipo de solução osmótica e o tempo de imersão. As soluções de sacarose a $0,3 \mathrm{~g} \mathrm{~mL}^{-1}+$ sucralose $0,2 \mathrm{~g} \mathrm{~L}^{-1}$, de sacarose a $0,4 \mathrm{~g} \mathrm{~mL}^{-1} \mathrm{e}$ sacarose a $0,5 \mathrm{~g} \mathrm{~mL}^{-1}$ mostraram comportamento semelhante, resultando em ganho de sólidos entre 4,7 e $6,4 \%$, respectivamente (Tabela 4 ).

TABELA 4. Ganho de sólidos (\%) dos pedaços de goiaba em função do tempo de desidratação osmótica, em soluções de sacarose a $0,3 \mathrm{~g} \mathrm{~mL}^{-1}+$ sucralose $0,2 \mathrm{~g} \mathrm{~L}^{-1}$, de sacarose a 0,5 e $0,4 \mathrm{~g} \mathrm{~mL}^{-1}$ e de açúcar invertido sem diluição. Solids gain $(\boldsymbol{\delta})$ in guava pieces as a function of osmo-dehydration time in sucrose at $0.3 \mathrm{~g} \mathrm{~mL}^{-1}+$ sucralose at $0.2 \mathrm{~g} \mathrm{~mL}^{-1}$, in sucrose solutions at 0.5 and $0.4 \mathrm{~g} \mathrm{~mL}^{-1}$, in undiluted inverted sugar syrup.

\begin{tabular}{ccccc}
\hline \multirow{2}{*}{ Tempo $(\mathrm{h})$} & \multicolumn{4}{c}{ Ganho de Sólidos $(\%)$} \\
\cline { 2 - 5 } & ${\text { Sac } 0,3 \mathrm{~g} \mathrm{~mL}^{-1}+\text { Suc } 0,2 \mathrm{~g} \mathrm{~L}^{-1}}_{\mathrm{Sac}^{-1}, 4 \mathrm{~g} \mathrm{~mL}^{-1}}$ & $\mathrm{Sac}_{0,5 \mathrm{~g} \mathrm{~mL}^{-1}}$ & Açúcar Invertido \\
\hline 0,1 & 1,5 & 1,4 & 2,8 & 3,0 \\
0,3 & 2,0 & 1,4 & 3,3 & 3,9 \\
0,5 & 2,9 & 2,6 & 4,3 & 4,7 \\
0,8 & 2,8 & 2,5 & 4,1 & 5,4 \\
1,0 & 3,0 & 2,9 & 5,6 & 5,7 \\
1,5 & 3,7 & 3,2 & 6,2 & 6,5 \\
2,0 & 5,1 & 4,1 & 6,4 & 8,2 \\
\hline
\end{tabular}

Os valores mencionados estão de acordo com os obtidos por PANADÉS et al. (2006), que observaram que o ganho de sólidos na desidratação de pedaços de goiaba por $6 \mathrm{~h}$ em soluções de sacarose a $65^{\circ}$ Brix variou no intervalo entre $2,3 \leq \delta \leq 10,1$, para temperaturas entre 30 e $50{ }^{\circ} \mathrm{C}$ e grau de agitação de $100 \mathrm{~min}$. Por outro lado, DIONELLO et al. (2007) observaram efeito positivo significativo da concentração da solução de sacarose no ganho de sólidos durante a desidratação 
osmótica de fatias de abacaxi a $50{ }^{\circ} \mathrm{C}$, por $2 \mathrm{~h}$ a $60 \mathrm{~min}^{-1}$. KARATHANOS et al. (1995), na desidratação osmótica de maçãs em soluções de sacarose e glicose a 15; 30 e 45\%, observaram que as amostras desidratadas em glicose apresentaram maior ganho de sólidos que aquelas desidratadas em sacarose. Os resultados díspares entre os experimentos são devidos, possivelmente, às diferenças nas estruturas dos tecidos das frutas avaliadas nos diferentes experimentos.

\section{Redução de massa}

As médias da percentagem de redução de massa $(\mu)$ dos pedaços de goiaba entre as diferentes soluções osmóticas empregadas no processo, dentro de cada tempo, encontram-se na Tabela 5. A redução de massa das amostras durante a desidratação osmótica corresponde à diferença entre a perda de água e o ganho de sólidos $(\mu=\omega-\delta)$. Assim, a solução mais concentrada de sacarose, que apresentou maior perda de água, também resultou em maior ganho de sólidos, $\delta$ final $=6,4 \%$ (Tabela 4), culminando com redução de massa estatisticamente igual à das amostras desidratadas com sacarose $0,4 \mathrm{~g} \mathrm{~mL}^{-1}$ (Tabela 5).

Semelhantemente ao ocorrido com a variável perda de água, em todos os tempos, as amostras submetidas à solução de sacarose a $0,3 \mathrm{~g} \mathrm{~mL}^{-1}+$ sucralose $0,2 \mathrm{~g} \mathrm{~L}^{-1}$ apresentaram perda de água menor que as demais $(\omega=15,1 \%)$, implicando, no final do processo, redução de massa inferior a $10 \%$. Esse efeito mais pronunciado dos valores da perda de água sobre a redução de massa de amostras de goiaba também foi observado por PANADÉS et al. (2006); a redução de massa obtida por esses autores variou no intervalo $15,6 \% \leq \mu \leq 37,4 \%$ para amostras desidratadas entre $30 \mathrm{e}$ $50{ }^{\circ} \mathrm{C}$ em solução de sacarose a $65^{\circ}$ Brix, por $3 \mathrm{~h}$. Os menores valores de $\mu$ obtidos no presente trabalho resultaram do emprego de soluções menos concentradas de sacarose ( 26 a 40 Brix).

TABELA 5. Redução de massa (\%) dos pedaços de goiaba em função do tempo de desidratação osmótica, em soluções de sacarose a $0,3 \mathrm{~g} \mathrm{~mL}^{-1}+$ sucralose $0,2 \mathrm{~g} \mathrm{~L}^{-1}$, de sacarose a 0,5 e $0,4 \mathrm{~g} \mathrm{~mL}^{-1}$ e xarope de açúcar invertido sem diluição. Mass reduction $(\mu)$ in guava pieces as a function of osmo-dehydration time in sucrose at $0.3 \mathrm{~g} \mathrm{~mL}^{-1}+$ sucralose at $0.2 \mathrm{~g} \mathrm{~mL}^{-1}$, in sucrose solutions at 0.5 and $0.4 \mathrm{~g} \mathrm{~mL}^{-1}$ and in undiluted inverted sugar syrup.

\begin{tabular}{ccccc}
\hline \multirow{2}{*}{ Tempo (h) } & \multicolumn{4}{c}{ Redução de Massa $(\%)$} \\
\cline { 2 - 4 } & Sac 0,3 $\mathrm{g} \mathrm{mL}^{-1}+$ Suc $0,2 \mathrm{~g} \mathrm{~L}^{-1}$ & ${\text { Sac } 0,4 \mathrm{~g} \mathrm{~mL}^{-1}}$ & ${\text { Sac } 0,5 \mathrm{~g} \mathrm{~mL}^{-1}}$ & Açúcar Invertido \\
\hline 0,1 & $3,13 \mathrm{~B}$ & $6,48 \mathrm{~A}$ & $6,68 \mathrm{~A}$ & $9,78 \mathrm{~A}$ \\
0,3 & $4,45 \mathrm{C}$ & $9,93 \mathrm{~B}$ & $10,93 \mathrm{~B}$ & $14,98 \mathrm{~A}$ \\
0,5 & $6,80 \mathrm{C}$ & $12,51 \mathrm{~B}$ & $14,17 \mathrm{~B}$ & $19,09 \mathrm{~A}$ \\
0,8 & $7,35 \mathrm{C}$ & $15,07 \mathrm{~B}$ & $15,92 \mathrm{~B}$ & $22,50 \mathrm{~A}$ \\
1,0 & $8,23 \mathrm{C}$ & $16,49 \mathrm{~B}$ & $18,00 \mathrm{~B}$ & $27,00 \mathrm{~A}$ \\
1,5 & $9,12 \mathrm{C}$ & $19,76 \mathrm{~B}$ & $19,80 \mathrm{~B}$ & $30,80 \mathrm{~A}$ \\
2,0 & $9,99 \mathrm{C}$ & $22,12 \mathrm{~B}$ & $21,95 \mathrm{~B}$ & $34,29 \mathrm{~A}$ \\
\hline
\end{tabular}

Valores seguidos de mesma letra maiúscula na linha não diferem entre si, a $5 \%$ de probabilidade, pelo teste de Tukey.

Com exceção do tempo de $0,1 \mathrm{~h}$, a solução de açúcar invertido provocou perda de água superior à dos demais tratamentos (Figuras 1 e 2), possibilitando redução de massa de 34,29\%, valor superior aos obtidos nas demais condições de processamento. Esse valor de $\mu$, obtido em solução de $64^{\circ}$ Brix, é próximo do valor máximo relatado por PANADÉS et al. (2006), $\mu=37,4 \%$, que foi obtido para a solução de $65^{\circ}$ Brix. Ao contrário do verificado no presente trabalho, DIONELLO et al. (2007) observaram que, na desidratação de fatias de abacaxi a $50{ }^{\circ} \mathrm{C}$ em xarope de açúcar invertido sem diluição, a redução de massa foi geralmente menor que nos tratamentos empregando soluções de sacarose menos concentradas. Argumentou-se que, a essa temperatura, aliada à alta concentração da solução $\left(70^{\circ}\right.$ Brix $)$, pode ter ocorrido maior facilidade para a impregnação de sólidos nas camadas superficiais, dificultando, assim, a perda de massa. Além disso, outros fatores relevantes seriam o valor elevado de massa específica $\left(1,36 \mathrm{~g} \mathrm{~cm}^{-3}\right)$ e da 
viscosidade da solução de açúcar invertido, em comparação com as demais soluções, que são menos densas e viscosas e apresentam menor teor de SST.

Para evitar grandes alterações no perfil sensorial e nutricional do produto, a eficiência do processo de desidratação osmótica pode ser avaliada pela ocorrência de maior perda de água e menor ganho de sólidos (LAZARIDES, 1994; KHOYI \& HESARI, 2006). De acordo com esse critério, o tratamento com solução de sacarose a $0,4 \mathrm{~g} \mathrm{~mL}^{-1}$ apresentou o melhor desempenho, pois mostrou-se semelhante àquele com sacarose a $0,5 \mathrm{~g} \mathrm{~mL}^{-1}$ no que diz respeito à perda de água (Figura 1) e similar ao de sacarose a $0,3 \mathrm{~g} \mathrm{~mL}^{-1}+$ sucralose $0,2 \mathrm{~g} \mathrm{~L}^{-1}$ em relação ao ganho de sólidos (Figura 2), culminando, assim, com redução de massa também similar à sacarose $0,5 \mathrm{~g} \mathrm{~mL}^{-1}$ (Figura 3). Afora isso, a solução de sacarose a $0,4 \mathrm{~g} \mathrm{~mL}^{-1}$, em relação à mais concentrada, proporcionaria, também, economia significativa de matéria-prima (sacarose) e a consequente redução do custo de produção.

\section{CONCLUSÕES}

A solução de açúcar invertido promoveu maior perda de água e redução de massa nas amostras de goiaba submetidas à desidratação osmótica. $\mathrm{O}$ melhor desempenho foi obtido para o tratamento em solução de sacarose a $0,4 \mathrm{~g} \mathrm{~mL}^{-1}$, com perda de água e redução de massa semelhantes aos valores obtidos na imersão em solução de sacarose a $0,5 \mathrm{~g} \mathrm{~mL}^{-1}$ e ganho de sólidos similar ao observado em solução de sacarose a $0,3 \mathrm{~g} \mathrm{~mL}^{-1}$.

\section{AGRADECIMENTOS}

Ao CNPq, FAPERJ, FINEP e International Foundation for Science (IFS), pelo apoio financeiro, e às empresas Attivos Magisttrais e Dulcini S.A. e à GOIACAM (Cooperativa de Produtores de Goiaba de Cachoeiras de Macacu), pelo apoio técnico.

\section{REFERÊNCIAS}

ALI, Z.M.; LAZAN, H. Guava. In: MITRA, S.K. Postharvest physiology and storage of tropical and subtropical fruits. New York: CAB International, 2001. p.146-165.

ANDRADE, S.A.C.; NETO, B.B.; NÓBREGA, A.C.; AZOUBEL, P.M.; GUERRA, N.B.

Evaluation of water and sucrose diffusion coefficients during osmotic dehydration of jenipapo (Genipa americana L.). Journal of Food Engineering, Amsterdam, v.78, n.2, p.551-555, 2007.

AOAC. ASSOCIATION OF OFFICIAL ANALYTICAL CHEMISTS. Method 932.12: Solids (soluble) in fruits and fruit products: Refractometer method: Final Action 1980. Arlington, 1990a.

AOAC. ASSOCIATION OF OFFICIAL ANALYTICAL CHEMISTS. Method 934.06: Moisture in dried fruits: Final action. Arlington, 1990b.

AZZOLINI, M.; JACOMINO, A.P.; BRON, I.U. Índices para avaliar qualidade pós-colheita de goiabas em diferentes estádios de maturação. Pesquisa Agropecuária Brasileira, Brasília, v.39, n.2, p.139-145, 2004.

BRUNINI, M.A.; OLIVEIRA, A.L.; VARANDA, D.B. Avaliação da qualidade de polpa de goiaba 'Paluma' armazenada a $-20{ }^{\circ} \mathrm{C}$. Revista Brasileira de Fruticultura, Jaboticabal, v.25, n.3, p.394396, 2003.

DHINGRA, D.; SINGH, J.; PATIL, R.T.; UPPAL, D.S. Osmotic dehydration of fruits and vegetables: A review. Journal of Food Science and Technology, Mysore, v.45, n.3, p.209-217, 2008.

DIONELLO, R.G.; BERBERT, P.A.; MOLINA, M.A.B.; VIANA, A.P.; CARLESSO, V.O.; QUEIROZ, V.A.V. Desidratação por imersão-impregnação de abacaxi em soluções de sacarose e em xarope de açúcar invertido. Ciência e Tecnologia de Alimentos, Campinas, v.27, n.4, p.787-792, 2007. 
FERRARI, C.C.; RODRIGUES, L.K.; TONON, R.V.; HUBINGER, M.D. Cinética de transferência de massa de melão desidratado osmoticamente em soluções de sacarose e maltose. Ciência e Tecnologia de Alimentos, Campinas, v.25, n.3, p.564-570, 2005.

KARATHANOS, V.T.; KOSTAROPOULOS, A.E.; SARAVACOS, G.D. Air-drying kinetics of osmotically dehydrated fruits. Drying Technology, London, v.13, n.5-7, p.1.503-1.521, 1995.

KHOYI, M.R.; HESARI, J. Osmotic dehydration kinetics of apricot using sucrose solution. Journal of Food Engineering, Amsterdam, v.78, n.4, p.1.355-1.360, 2007.

LAZARIDES, H.N. Osmotic preconcentration: Developments and prospects. In: SINGH, R.P.; OLIVEIRA, F.A.R. Minimal processing of foods and process optimization: An Interface. Boca Raton: CRC Press, 1994. p.73-85.

PANADÉS, G.; CASTRO, D.; CHIRALT, A.; FITO, P.; NUÑEZ, M.; JIMENEZ, R. Mass transfer mechanisms occurring in osmotic dehydration of guava. Journal of Food Engineering, Amsterdam, v.87, n.3, p.386-390, 2008.

PANADÉS, G.; FITO, P.; AGUIAR, Y.; VILLAVICENCIO, M.N.; ACOSTA, V. Osmotic dehydration of guava: Influence of operating parameters on process kinetics. Journal of Food Engineering, Amsterdam, v.72, n.4, p.383-389, 2006.

PARK, K.J.; BIN, A.; BROD, F.P.R. Drying of pear d'Anjou with and without osmotic dehydration. Journal of Food Engineering, Amsterdam, v.56, n.1, p.97-103, 2003.

PEREIRA, L.M.; RODRIGUES, A.C.C.; SARANTOPOULOS, C.I.G.L.; JUNQUEIRA, V.C.A.; CUNHA, R.L.; HUBINGER, M.D. Influence of modified atmosphere packaging and osmotic dehydration on the quality maintenance of minimally processed guavas. Journal of Food Science, Chicago, v.69, n.4, p.E172-E177, 2004.

PINO, J.A.; PANADÉS, G.; FITO, P.; CHIRALT, A.; ORTEGA, A. Influence of osmotic dehydration on the volatile profile of guava fruits. Journal of Food Quality, Ames, v.31, n.3, p.281294, 2008.

QUEIROZ, V.A.V.; BERBERT, P.A.; MOLINA, M.A.B.; GRAVINA, G.A.; QUEIROZ, L.R.; DELIZA, R. Desidratação por imersão-impregnação e secagem por convecção de goiaba. Pesquisa Agropecuária Brasileira, Brasília, v.2, n.10, p.1.479-1.486, 2007.

QUEIROZ, V.A.V.; BERBERT, P.A.; MOLINA, M.A.B.; GRAVINA, G.A.; QUEIROZ, L.R.; SILVA, J.A. Qualidade nutricional de goiabas submetidas aos processos de desidratação por imersão-impregnação e secagem complementar por convecção. Ciência e Tecnologia de Alimentos, Campinas, v.28, n.2, p.329-340, 2008.

RIVA, M.; CAMPOLONGO, S.; LEVA, A.A.; MAESTRELLI, A.; TORREGGIANI, D. Structureproperty relationships in osmo-air-dehydrated apricot cubes. Food Research International, Amsterdam, v.38, n.5, p.533-542, 2005.

SILVEIRA, E.T.F.; RAHMAN, M.S.; BUCKLE, K.A. Osmotic dehydration of pineapple: kinetics and product quality. Food Research International, Amsterdam, v.29, n.3-4, p.227-233, 1996.

TACO. Tabela brasileira de composição de alimentos. 2.ed. Campinas: NEPA-UNICAMP, 2006. v.2. $113 \mathrm{p}$.

TORREGGIANI, D.; BERTOLO, G. High-quality fruit and vegetable products using combined processes. In: FITO, P.; CHIRALT, A.; BARAT, J.M.; SPIESS, W.E.L.; BEHSNILIAN, D. Osmotic dehydration e vacuum impregnation. Lancaster: Technomic Publishing Company, 2001. p.3-9. 